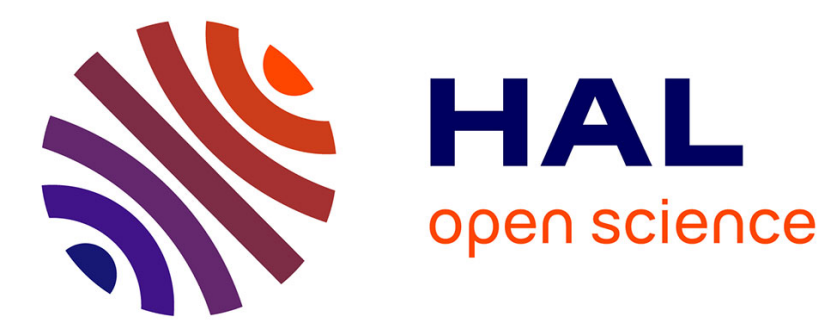

\title{
On a Simple Equilibrium with Heterogeneous Quasi-Hyperbolic Discounting Agents
}

\author{
Jean-Pierre Drugeon, Bertrand Wigniolle
}

\section{To cite this version:}

Jean-Pierre Drugeon, Bertrand Wigniolle. On a Simple Equilibrium with Heterogeneous QuasiHyperbolic Discounting Agents. 2018. halshs-01962004

\section{HAL Id: halshs-01962004 \\ https://shs.hal.science/halshs-01962004}

Preprint submitted on 20 Dec 2018

HAL is a multi-disciplinary open access archive for the deposit and dissemination of scientific research documents, whether they are published or not. The documents may come from teaching and research institutions in France or abroad, or from public or private research centers.
L'archive ouverte pluridisciplinaire HAL, est destinée au dépôt et à la diffusion de documents scientifiques de niveau recherche, publiés ou non, émanant des établissements d'enseignement et de recherche français ou étrangers, des laboratoires publics ou privés. 


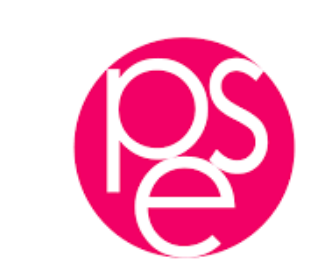

PARISSCHOOLOFECONOMICS
ECOLED'ECONOMIEDEPARIS

WORKING PAPER Nº $2018-61$

On a Simple Equilibrium with Heterogeneous Quasi-Hyperbolic Discounting Agents

\author{
Jean-Pierre Drugeon \\ Bertrand Wigniolle
}

JEL Codes: E32, C62

Keywords : Heterogeneities, quasi-hyperbolic discounting, linear decision rules
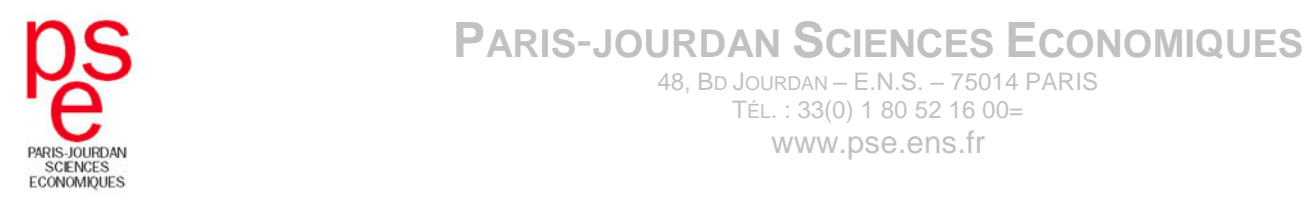


\title{
On a Simple Equilibrium With HeterogeneOUS QuASI-HyPerbolic Discounting Agents*
}

\author{
JEAN-PierRe Drugeon ${ }^{\dagger} \&$ Bertrand Wigniolle ${ }^{\ddagger}$
}

$4^{\text {th }}$ December 2018

\footnotetext{
${ }^{*}$ This research was completed thanks to the Novo Tempus research grant, ANR-12-BSH1-ooo7, Program BSH1-2012, and the Fire research grant, ANR-15-CE33-0oo1o.

${ }^{\dagger}$ Paris School of Economics and Centre National de la Recherche Scientifique.

¥Paris School of Economics and University Paris 1 Panthéon-Sorbonne.
} 


\begin{abstract}
This article considers the long-run equilibrium distribution of an economy populated by heterogenous and present biased quasi-hyperbolic discounting agents. In a first configuration with logarithmic utility functions and Cobb-Douglas production technologies, this article establishes the existence and the uniqueness of the equilibrium: only one agent, determined by the highest value of a coefficient building from both the degree of present bias and the rate of discount, will have a positive long-run consumption and a positive long-run wealth. A second configuration with constant elasticities of substitution utilities and linear production technologies is then considered. This article similarly establishes the existence and the uniqueness of the equilibrium. There is generically a unique agent with the highest growth rate for his consumption and his wealth. This agent is determined by both preferences and technology parameters and may change following a technological shock.
\end{abstract}

KEYWORDS: Heterogeneities, quasi-hyperbolic discounting, linear decision rules.

JEL Classification: E32, C62.

Contents

1 INTRODUCTION 1

2 The Model 2

3 An Economy with Logarithmic Preferences and a Cobb-Douglas ProDUCTION TECHNOLOGY

4 An Economy with C.E.S. Utility and a Linear Production Technology $\mathbf{5}$

4.1 The Sophisticated Agent $\ldots \ldots \ldots \ldots \ldots \ldots$

4.2 The Naïve Agent $\ldots \ldots \ldots \ldots \ldots \ldots \ldots \ldots \ldots \ldots \ldots \ldots$

\begin{tabular}{l|c} 
A Proof of Lemma 3.1 & 8
\end{tabular}

B $\quad$ Proof of Lemma $3.2 \quad 9$

$\begin{array}{lll}\text { C Proof of Proposition } 3.1 & 11\end{array}$

$\begin{array}{lll}\text { D Proof of Lemma } 4.1 & 13\end{array}$

$\begin{array}{lll}\text { E } & \text { Proof of Proposition 4.1 } & 15\end{array}$

$\begin{array}{lll}\text { F } & \text { Proof of Proposition 4.2 } & 16\end{array}$

$\begin{array}{lrr}\text { G Proof of Proposition 4.3 } & \mathbf{1 7}\end{array}$ 


\section{INTRODUCTION}

The model with heterogenous agents that differ from their valuation of the future and their discount rate has motivated a large literature focused on the features of the associated inter temporal equilibrium. The Ramsey's Conjecture states that only the most patient amongst the agents would end up exhibiting positive consumption levels in the long run, all of the other agents being associated with nil consumption levels. It was rigorously proved by Bewley [4] who establishes that the long-run equilibrium assumes the following properties: the most patient amongst the agents holds the whole capital stock of the economy and assumes positive consumption levels. By contrast, all of the remaining agents have asymptotically nil consumption levels. It is also worth mentioning that the level of capital in this model corresponds to a modified golden rule defined from the discount factor of the most patient agent.

The assumption according to which agents would differ in their valuation of the future and their discounting rates has led to numerous further developments. Becker [1] was the first to reconsider this Ramsey's Conjecture under the assumption of imperfect financial markets - a non-negativity constraint is imposed on household capital holdings-. The long-run equilibrium is then associated with two classes of agents, namely the most patient capitalist agent who owns the whole capital stock of the economy, and the other more impatient workers agents who consume their current wages. Becker \& Foias [2] and Sorger [12] have later enriched this framework with strategic dimensions.

This article is interested in the status of the aforementioned Ramsey's Conjecture when agents have quasi-hyperbolic discounting and simultaneously differ by their discount rates and their degrees of present bias. Dating from the seminal contributions of Phelps \& Pollack [9], Laibson [8] and Frederick, Loewenstein \& O'Donoghue [6], the benchmark model for analyzing temporal inconsistencies got anchored on the quasi-hyperbolic discounting hypothesis and gained popularity through the so-called $\beta-\delta$ model, where $\delta \in] \mathrm{o}, 1[$ features the discount rate and $\beta<1$ features the present bias. Numerous experiments ${ }^{1}$ have indeed consistently illustrated how the quasi-hyperbolic discounting $\beta-\delta$ model could provide a more accurate description of actual choices and arbitrages over time than the canonical model as settled by Samuelson [11] and Ramsey [10]. A large number of studies (see Frederick, Loewenstein \& O'Donoghue [6]) have also ruled field or laboratory experiments in order to evaluate the $\beta$ and $\delta$ parameters. They all document a strong heterogeneity between the individuals regarding these parameters.

This article considers a simple competitive economy with heterogenous quasi-hyperbolic infinitely-lived agents. Agents preferences being temporally inconsistent, it is assumed that these agents build from a sequence of successive selves, their decisions resulting from a Nash equilibrium between such selves. This assumption is commonly referred as depicting a sophisticated behaviour. This is completed by a production side with a standard neoclassical production technology. While no explicit solution would be available in the general case, the argument of this article is specialized to specific parametric formulations that will allow closed forms for the strategies of the agents.

In a first configuration with logarithmic utility functions and Cobb-Douglas production

\footnotetext{
${ }^{1}$ See Thaler [13], Benzion, Rapoport \& Yagil [3], Chapman \& Elstein [5] or Frederick, Loewenstein O’Donoghue [6] for a general presentation.
} 
technologies, this article establishes the existence and the uniqueness of an equilibrium with heterogenous quasi-hyperbolic discounting agents. The properties of the long-run steady state remain close from the ones of the classical Ramsey model: only one agent will have a positive long-run consumption and a positive long-run wealth. That agent is however determined by the value of a coefficient where the two parameters $\beta$ and $\delta$ come into play and interact. The impatience of a given agent-a relatively weak value for $\delta$ - can be compensated by a relative low present bias-a relatively high value for $\beta$.

In a second configuration with constant elasticities of substitution utilities and linear production technologies, this article similarly establishes the existence and the uniqueness of an equilibrium with heterogenous quasi-hyperbolic discounting agents. This competitive equilibrium converges towards a steady growth rate for the capital stock. Interestingly, the consumptions of the agents are going to assume constant but distinct equilibrium growth rates. These individuals rates of growth are not only determined by the two parameters $\beta$ and $\delta$ but also by the constant elasticity of substitution of the utility and the constant productivity of the capital stock. There is generically a unique agent with the highest growth rate for his consumption and his wealth. This agent determines the long-run growth rate for the capital stock of this economy. The consumptions of the other agents become negligible in the long run when compared with the one of this agent. It is further proved that a given perturbation in the value of the constant productivity of the capital stock parameter may end up in modifying the ranking associated with the individual consumption growth rates between two distinct individuals. The case of naïve agents is finally analysed: this is shown to favour (detriment) capital accumulation when the elasticity of substitution is greater (lower) than one

Section 2 introduces a general environment with present biased quasi-hyperbolic discounting agents. An environment with logarithmic utilities and Cobb-Douglas production technologies is considered in Section 3. Another environment with CES utilities and linear production technologies is analyzed in Section 4. All proofs and technicalities are gathered in an final appendix.

\section{THE MODEL}

Time is discrete. Consider a competitive economy with $n$ infinitely-lived agents indexed by $i \in\{1,2, \ldots, n\}$. Let $\left(c_{t}^{i}\right)_{t \in \mathbb{N}}$ denote the consumption sequence of individual $i$, while $\left(w_{t}\right)_{t \in \mathbb{N}}$ and $\left(R_{t}\right)_{t \in \mathbb{N}}$ respectively stand for the wage and gross interest rates sequences he faces with, for $R_{t}=1+r_{t}$ and $r_{t}$ the interest rate.

Letting $a_{t}^{i}$ denote the capital stock held at date $t \in \mathbb{N}$ by agent $i$, his budget constraint for date $t$ is expressed as follows:

$$
a_{t+1}^{i}=R_{t} a_{t}^{i}+w_{t}-c_{t}^{i} .
$$

Agents are further subject to an inter-temporal budget constraint defined over their lifespan. Assuming that $\sum_{t=0}^{+\infty}\left(w_{t} / \prod_{\tau=0}^{t} R_{\tau}\right)<+\infty$, the actualised wealth of agent $i$, denoted as $W_{t}^{i}$, is defined by:

$$
W_{t}^{i}=R_{t} a_{t}^{i}+w_{t}+\sum_{\tau=t+1}^{+\infty} \frac{w_{\tau}}{\prod_{j=t+1}^{\tau} R_{j}},
$$


while the inter-temporal budget constraint can be expressed, for every $t \in \mathbb{N}$, as:

$$
\begin{aligned}
& W_{t+1}^{i}=R_{t+1}\left(W_{t}^{i}-c_{t}^{i}\right), \\
& \text { with } W_{t}^{i} \geqslant 0 \text { for every } t \geqslant 0 .
\end{aligned}
$$

The preferences of the agents are associated with quasi-geometric discounting. For a given agent $i \in\{1,2, \ldots, n\}$, his self at time $t \in \mathbb{N}$ ranks consumption sequences according to:

$$
\left.u\left(c_{t}^{i}\right)+\beta_{i}\left[\sum_{\tau=1}^{+\infty}\left(\delta_{i}\right)^{\tau} u\left(c_{t+\tau}^{i}\right)\right], \beta_{i}>\mathrm{o}, \delta_{i} \in\right] \mathrm{o}, \mathrm{1}[.
$$

The decisions of agent $i \in\{1,2, \ldots, n\}$ stem from the Nash equilibrium of a strategic game between the successive selves - a Markov equilibrium, also commonly referred as a sophisticated behaviour. Within an infinite horizon setup, it was established by Krusell \& Smith [7] that it is possible to build a multiplicity of equilibrium solutions between the different selves. In opposition to this, for a finite horizon environment, the solution obtained by backward induction is unique. This article will retain the infinite horizon solution that is obtained as the limit of the finite horizon one. This enables to select a specific solution amongst the multiple admissible infinite horizon ones.

This article willl consider two separate configurations, i.e., a first one with a logarithmic utility function and a Cobb-Douglas production technology and a second one with an isoelastic utility function and a linear production technology. As this will be established in the remaining exposition, both of these configurations will be associated with linear decision rules for the consumers $i \in\{1, \ldots, n\}$ :

$$
c_{t}^{i}=\lambda_{i} W_{t}^{i}
$$

with $\lambda_{i} \in[0,1]$. Without loss of generality, agents will henceforward be ranked according to $\lambda_{1} \leqslant \lambda_{2} \leqslant \ldots \leqslant \lambda_{n}$, where it is assumed that $\lambda_{1}<\lambda_{2}$. The solution that is obtained as the limit of the finite horizon game assumes well-behaved regularity properties — they are indeed linear. In opposition to this, the strategies raised by Krusell \& Smith [7] were not smooth.

The economy under consideration also integates a competitive representative firm with a production technology $F(K, L)$ that satisfies:

Assumption T. 1. $F(K, L)$ is a function of class $\mathscr{C}^{2}$, homogeneous of degree one, increases with $K$ as $D_{K} F(K, 1)>0$, and is concave.

$K_{t}$ being the capital stock of period $t$ and $L_{t}$ the quantity of labour of that same period $t$, the capital stock being assumed to fully depreciate on use within period $t$. All individuals inelastically offer $1 / n$ units of labour, so that the global labour supply amounts to 1 . The competitive wage rate and the competitive interest factor are thus available at date $t \in \mathbb{N}$ as:

$$
\begin{aligned}
w_{t} & =\frac{1}{n} D_{L} F\left(K_{t}, 1\right), \\
R_{t} & =D_{K} F\left(K_{t}, 1\right) .
\end{aligned}
$$

An equilibrium is then characterised as follows: 
DefinITION 2.1. A competitive equilibrium of the economy is characterised by a sequence $\left(W_{t}^{i}, K_{t}\right)_{t \in \mathbb{N}}$ such that, for every $t \in \mathbb{N}$ and every $i \in\{1,2, \ldots, n\}$ :

$$
\begin{aligned}
& W_{t+1}^{i}=R_{t+1}\left(1-\lambda_{i}\right) W_{t}^{i}, \\
& K_{t+1}=F\left(K_{t}, 1\right)-\sum_{i=1}^{n} \lambda_{i} W_{t}^{i},
\end{aligned}
$$

starting from initial conditions $W_{\mathrm{o}}^{i}=R_{0} a_{\mathrm{o}}^{i}+w_{0}+\sum_{t=1}^{+\infty}\left[w_{t} / \prod_{\tau=1}^{t} R_{\tau}\right]$ with $a_{\mathrm{o}}^{i}$ given such that $\sum_{i=1}^{n} a_{\mathrm{o}}^{i}=K_{\mathrm{o}}$ and $\left(w_{t}, R_{t}\right)$ given by (4a) and $(4 b)$.

Equation $(5 a)$ is obtained from the inter-temporal budget constraint (1), respectively replacing $R_{t}$ by Equation $(4 b)$ and $c_{t}^{i}$ by Equation (3). Similarly, equation $(5 b)$ derives from the integration of the consumption decision rules (3) into the resource constraint of the economy.

\section{An Economy with Logarithmic Preferences and a Cobb-Douglas Production Technology}

This section will consider a configuration where $u(c)=\ln (c)$ and $F\left(K_{t}, L_{t}\right)=\left(K_{t}\right)^{\alpha}\left(L_{t}\right)^{1-\alpha}$, $\alpha \in[0,1]$, that in turn implies equilibrium returns of $w_{t}=(1 / n)(1-\alpha)\left(K_{t}\right)^{\alpha}$ and $R_{t}=$ $\alpha\left(K_{t}\right)^{\alpha-1}$.

Lemma 3.1. For $u(c)=\ln (c)$ and $F\left(K_{t}, L_{t}\right)=\left(K_{t}\right)^{\alpha}\left(L_{t}\right)^{1-\alpha}, \alpha \in[0,1]$, the decision rules of the consumers resulting from a Nash equilibrium between the successive selves list as:

$$
\begin{aligned}
c_{t}^{i} & =\lambda_{i} W_{t}^{i}, \\
\lambda_{i} & =\frac{1-\delta_{i}}{1-\delta_{i}+\beta_{i} \delta_{i}}
\end{aligned}
$$

LEMMA 3.2. Up to its existence, a competitive equilibrium of the economy is characterised, for every $i \in\{1,2, \ldots, n\}, b y$ :

$$
\begin{aligned}
& W_{t}^{i}=\frac{W_{\mathrm{o}}^{i}\left[\alpha\left(1-\lambda_{i}\right)\right]^{t}}{\sum_{j=1}^{n} \lambda_{j} W_{\mathrm{o}}^{j}\left[\alpha\left(1-\lambda_{j}\right)\right]^{t} /\left[1-\alpha\left(1-\lambda_{j}\right)\right]}, \\
& K_{t+1}=\left(K_{t}\right)^{\alpha}\left(1-\gamma_{t}\right), \\
& \text { with } \quad \gamma_{t}:=\frac{\sum_{i=1}^{n} \lambda_{i} W_{\mathrm{o}}^{i}\left[\alpha\left(1-\lambda_{i}\right)\right]^{t}}{\sum_{i=1}^{n} \lambda_{i} W_{\mathrm{o}}^{i}\left[\alpha\left(1-\lambda_{i}\right)\right]^{t} /\left[1-\alpha\left(1-\lambda_{i}\right)\right]},
\end{aligned}
$$

starting from initial conditions $W_{\mathrm{o}}^{i}=R_{0} a_{\mathrm{o}}^{i}+w_{\mathrm{o}}+\sum_{t=1}^{+\infty}\left[w_{t} / \prod_{\tau=1}^{t} R_{\tau}\right]$ with $a_{\mathrm{o}}^{i}$ given such that $\sum_{i=1}^{n} a_{\mathrm{o}}^{i}=K_{\mathrm{o}}$ and $\left(w_{t}, R_{t}\right)$ given by $(4 a)$ and $(4 b)$.

Proposition 3.1. - Consider the above economy:

(i) if $a_{\mathrm{o}}^{i} \geqslant \mathrm{o}$ for every $i \in\{1, \ldots, n\}$, there exists a unique competitive equilibrium;

(ii) the competitive equilibrium converges to a steady state characterised by the following features:

$\mathrm{a} /$ the capital stock converges to a modified golden rule like steady state with a value $\overline{\mathrm{K}}$ that corresponds to the only preferences parameters of agent 1 through the parameter $\lambda_{1}$ :

$$
\alpha(\bar{K})^{\alpha-1}=\frac{1}{1-\lambda_{1}} ;
$$


$\mathrm{b} /$ agent 1 is the only agent with a long-run positive inter-temporal wealth $W^{1}=(\bar{K})^{\alpha}[1-$ $\left.\alpha\left(1-\lambda_{1}\right)\right] / \lambda_{1}$. By contrast with this, the wealth of all the other agents is to converge to o;

c/ agent 1 is the only agent with a long-run positive consumption $\bar{c}^{1}=(\bar{K})^{\alpha}\left[1-\alpha\left(1-\lambda_{1}\right)\right]$. By contrast with this, the consumption of all other agents is to converge to 0.

This proposition hence yields a range of conclusions which are close to the ones of the classical Ramsey model: only one agent will have a positive long-run consumption and a positive long-run wealth. That agent is however determined by the value of the coefficient $\lambda_{i}$ where the two parameters $\beta_{i}$ and $\delta_{i}$ come into play and interact. The impatience of a given agent-a relatively weak value for $\delta_{i}$ - can be compensated by a relative low present bias-a relatively high value for $\beta_{i}$.

\section{An Economy with C.E.S. Utility AND a Linear Production Technology}

\subsection{The Sophisticated Agent}

Preferences are modified to a representation $u(c)=c^{1-1 / \sigma} /(1-1 / \sigma), \sigma \neq 1$, while the production technology is changed to $F\left(K_{t}, L_{t}\right)=A K_{t}, A>0$. It immediately follows that, for every $t \in \mathbb{N}, R_{t}=A, w_{t}=0$. It is similarly derived that $W_{0}^{i}=A a_{0}^{i}$. An extra assumption is needed in order to maintain a bounded utility for the agents:

Assumption PT. $1 . \forall i \in\{1,2, \ldots, n\}, \delta_{i} A^{1-1 / \sigma}<1$.

LemMa 4.1. For $u(c)=c^{1-\sigma} /(1-\sigma), \sigma \neq 1$ and $F\left(K_{t}, L_{t}\right)=A K_{t}, A>0$, the decision rules of the consumers resulting from a Nash equilibrium between the successive selves list as:

$$
c_{t}^{i}=\lambda_{i} W_{t}^{i}
$$

where the coefficient $\lambda_{i}$ is defined as the solution of the following equation:

$$
\left[A\left(1-\lambda_{i}\right)\right]^{1 / \sigma}=\delta_{i} A\left[1-\lambda_{i}\left(1-\beta_{i}\right)\right] .
$$

Interestingly, in equation (8), the coefficient $\lambda_{i}$ not only depends on the preferences parameters $\sigma, \delta_{i}$ and $\beta_{i}$ but also on the technological parameter $A$.

Proposition 4.1. - Consider the above economy:

(i) if $a_{\mathrm{o}}^{i}>$ o for every $i \in\{1, \ldots, n\}$, there exists a unique competitive equilibrium characterised, for every $t \in \mathbb{N}$ and for every $i \in\{1, \ldots, n\}$, by:

$$
\begin{aligned}
& W_{t}^{i}=A^{t+1}\left(1-\lambda_{i}\right)^{t} a_{\mathrm{o}}^{i}, \\
& c_{t}^{i}=\lambda_{i} A^{t+1}\left(1-\lambda_{i}\right)^{t} a_{\mathrm{o}}^{i},
\end{aligned}
$$

and by

$$
\begin{aligned}
& K_{t+1}=A K_{t}\left(1-\gamma_{t}\right), \\
& \text { for } \gamma_{t}=\frac{\sum_{i=1}^{n} \lambda_{i}\left(1-\lambda_{i}\right)^{t} a_{\mathrm{o}}^{i}}{\sum_{i=1}^{n}\left(1-\lambda_{i}\right)^{t} a_{\mathrm{o}}^{i}} ;
\end{aligned}
$$


(ii) the competitive equilibrium converges towards a stationary equilibrium characterised by a growth factor of the capital stock $K_{t+1} / K_{t}$ that converges towards a limit $A\left(1-\lambda_{1}\right)$.

Within this environment and as made clear by Proposition 4.1, the consumptions of the individual agents are going to assume constant but distinct equilibrium growth rates. All of the consumptions of the agents $i \geqslant 2$ would hence become negligible in the long run when compared with the one of agent $i=1$ who is associated with the lowest value for $\lambda_{i}$, the long-run growth rate of the capital stock being further exactly the same as the consumption growth rate of that later agent. From Equation (8), the values of $\lambda_{i}$ are to be jointly determined by $A, \sigma, \delta_{i}$ and $\beta_{i}$ for any of the individuals $i \in\{1, \ldots, n\}$. The following proposition then establishes how a given perturbation in the value of $A$ may end up in modifying the ranking associated with the coefficients $\lambda_{i}$ for two distinct individuals.

Proposition 4.2. - Let $\sigma>1$ and assume that two individuals $i \in\{1,2\}$ are characterised by parameters $\delta_{i}$ and $\beta_{i}$ such that:

$$
\delta_{1}<\delta_{2}, \quad \beta_{1}>\beta_{2} \quad \text { and } \quad \beta_{1} \delta_{1}>\beta_{2} \delta_{2} .
$$

There then exists some critical value for A, labelled $\hat{A}$, such that:

$$
\begin{cases}\text { for } A<\hat{A}, & \lambda_{1}<\lambda_{2} \\ \text { for } A>\hat{A}, & \lambda_{1}>\lambda_{2} .\end{cases}
$$

This proposition shows how a technological shock-a change in the parameter $A$-may result in a change in the identity of the agent who is determining the long-run growth rate of the economy.

\subsection{The NaÏve Agent}

It has up to now been assumed that agents have sophisticated behaviours. An alternative assumption however states as the hypothesis of naïve agents. A naïve $t$-self undertakes its decision at period $t$ as if it could also select all of its future decisions. For the logarithmic case of Section 3, naïve and sophisticated behaviours lead to the same decision rules. Oppositely and under the current assumption of CES utility functions, naïve and sophisticated behaviours result in distinct optimal strategies. This section is aimed at comparing these two strategies.

Assume instead that any self determines his behaviour for his decision period as if he could equally undertake the whole range of future decisions. This also corresponds to the solution for a self with a perfect commitment capacity. Henceforth labelling as naïve such a range of behaviours, it is established :

Proposition 4.3. For $u(c)=c^{1-\sigma} /(1-\sigma), \sigma \neq 1$ and $F\left(K_{t}, L_{t}\right)=A K_{t}, A>0$, consider a naïve agent:

(i) His decision rule is given by:

$$
\begin{aligned}
c_{t} & =\lambda_{i}^{n} W_{t}^{i}, \\
\lambda_{i}^{n} & =\frac{1-\left(\delta_{i}\right)^{\sigma} A^{\sigma-1}}{1-\left(\delta_{i}\right)^{\sigma} A^{\sigma-1}\left(1-\left(\beta_{i}\right)^{\sigma}\right)} .
\end{aligned}
$$


(ii) For $\beta_{i}<1, \lambda_{i}<\lambda_{i}^{n} \Longleftrightarrow \sigma<1$ and $\lambda_{i}>\lambda_{i}^{n} \Longleftrightarrow \sigma>1$.

(iii) Consider an economy populated by two agents with the same $(\beta, \delta)$ parameters and assume that one is sophisticated while the other one is naïve. The naïve agent accumulates more (less) capital that the sophisticated one when $\sigma>1(\sigma<1)$.

Consider an economy populated with two agents with the same $(\beta, \delta)$ parameters, the first being sophisticated while the second is naïve. For $\sigma>1(\sigma<1)$, the naïve (sophisticated) agent determines the long-run rate of growth.

\section{REFERENCES}

[1] Becker, R. On the Long-Run Stationary Competitive Equilibrium in a Simple Dynamic Model of Equilibrium with Heterogeneous Households. Quarterly Journal of Economics 95: 37582,1980 .

[2] Becker, R. \& C. Foias Strategic Ramsey equilibrium dynamics. Journal of Mathematical Economics 43: 318-346, 2007.

[3] Benzion, U., A. Rapoport \& J. Yagil Discount Rates Inferred from Decisions: An Experimental Study. Management science 35: 270-284, 1989.

[4] Bewley, T. An Integration of Equilibrium Theory and Turnpike Theory. Journal of Mathematical Economics 10: 233-267, 1982.

[5] Chapman, G. \& A. Elstein. Valuing the Future: Temporal Discounting of Health and Money. Medical Decision Making 15: 373-86, 1995.

[6] Frederick,S., G. Loewenstein \& T O’Donoghue. Time Discounting and Time Preference: A Critical Review. Journal of economic literature 40: 351-401, 2002.

[7] Krusell, P., \& A.S. Smith. Consumption-Savings Decisions with Quasi-Geometric Discounting. Econometrica 71: 365-375, 2003.

[8] Laibson, D. Golden Eggs and Hyperbolic Discounting. Quarterly Journal of Economics 112: 443-478, 1997 .

[9] Phelps, P., \& R. Pollack On Second-best National Saving and Game-Equilibrium Growth. Review of Economics Studies 35: 185-199, 1968.

[10] Ramsey, F. A Mathematical Theory of Savings. Economic Journal 38: 543-549, 1928.

[11] Samuelson, P. A Note on Measurement of Utility. Review of Economic Studies 4: 155-161, 1937.

[12] Sorger, G. On the Long-Run Distribution of Capital in the Ramsey Model. Journal of Economic Theory 105: 226-243, 2002.

[13] Thaler, R. Some Empirical Evidence On Dynamic Inconsistency. Economics Letters 8: 201$207,1981$. 


\section{A. Proof of Lemma 3.1}

Consider an agent with the preference parameters $(\beta, \delta)$. The game between selves is simplified by introducing the following variables:

$$
\begin{aligned}
X_{t} & =\frac{W_{t}}{\Pi_{t}} \\
x_{t} & =\frac{c_{t}}{\Pi_{t}}
\end{aligned}
$$

with $\Pi_{t}$ defined as:

$$
\Pi_{0}=1
$$$$
\text { for } t \geq 1, \Pi_{t}=\prod_{\tau=1}^{t} R_{\tau}
$$

The intertemporal budget constraint (1) becomes: $X_{t+1}=X_{t}-x_{t}$ with $x_{t} \leq X_{t}$. The objective functions of self $t(2)$ can be written as:

$$
\ln x_{t}+\beta \sum_{\tau=1}^{+\infty} \delta^{\tau} \ln x_{t+\tau}+H_{t}
$$

with $H_{t}$ some term that is exogenous for the agent.

Consider the game defined on a $T$ period horizon. The variables $X_{t}$ and $x_{t}$ are now denoted by $X_{t}^{T}$ and $x_{t}^{T}$. For $t \leq T$, the $t$-self objective function is:

$$
\ln x_{t}^{T}+\beta \sum_{\tau=1}^{T-t} \delta^{\tau} \ln x_{t+\tau}^{T}+H_{t}^{T}
$$

The game is solved by backward induction. As the objective function of all selves are homothetic, it is straightforward that the solution will take the following form:

$$
x_{t}^{T}=\lambda_{t}^{T} X_{t}^{T}
$$

It is first noticed that, for $t=T$, the solution is obvious with $\lambda_{T}^{T}=1$.

Then assume that the game has been solved till period $t+1$. One gets: $\forall \tau$ with $2 \leq \tau \leq T$,

$$
x_{t+\tau}^{T}=\lambda_{t+\tau}^{T}\left(1-\lambda_{t+\tau-1}^{T}\right) \cdots\left(1-\lambda_{t+1}^{T}\right) X_{t+1}^{T}
$$

and $x_{t+1}^{T}=\lambda_{t+1}^{T} X_{t+1}^{T}$. Replacing in the $t$-self objective function, we get:

$$
\ln x_{t}^{T}+\beta\left(\sum_{\tau=1}^{T} \delta^{\tau}\right) \ln X_{t+1}^{T}+G_{t}^{T}
$$

with $G_{t}^{T}$ some term that is exogenous for the self. Finally, the program of self $t$ can be written as:

$$
\begin{gathered}
\max _{\left(x_{t}^{T}, X_{t+1}^{T}\right)}\left[\ln x_{t}^{T}+\beta\left(\sum_{\tau=1}^{T} \delta^{\tau}\right) \ln X_{t+1}^{T}\right] \\
\text { s.t. } x_{t}^{T}+X_{t+1}^{T}=X_{t}^{T}
\end{gathered}
$$

The solution is: $x_{t}^{T}=\lambda_{t}^{T} X_{t}^{T}, X_{t+1}^{T}=\left(1-\lambda_{t}^{T}\right) X_{t}^{T}$ with

$$
\lambda_{t}^{T}=\frac{1}{1+\beta \sum_{\tau=1}^{T} \delta^{\tau}}
$$

For $T \rightarrow+\infty$, the limit strategy is derives as:

$$
\lim _{T \rightarrow+\infty} \lambda_{t}^{T}=\lambda=\frac{1}{1+\beta \delta /(1-\delta)},
$$

that completes the statement. 


\section{B. Proof of Lemma 3.2}

The argument of the proof revolves around a careful study of the dynamics of the system as described by Equations $\left(5^{a}\right)$ and $\left(5^{b}\right)$. Letting firstly the auxiliary variable $\chi_{t}$ be introduced as:

$$
\chi_{t}:=\frac{\left(K_{t}\right)^{\alpha}}{W_{t}^{1}} .
$$

From Equation $(5 a)$ considered for $i=1$, it is obtained that:

$$
W_{t+1}^{1}\left(K_{t+1}\right)^{1-\alpha}=\alpha\left(1-\lambda_{1}\right) W_{t}^{1} .
$$

Considering Equation $\left(5^{b}\right)$ and dividing each member by the corresponding one in Equation (A.2), it is derived that:

$$
\begin{aligned}
& \frac{\left(K_{t+1}\right)^{\alpha}}{W_{t+1}^{1}}=\frac{\left(K_{t}\right)^{\alpha}}{\alpha\left(1-\lambda_{1}\right) W_{t}^{1}}-\sum_{i=1}^{n} \frac{\lambda_{i}}{\alpha\left(1-\lambda_{1}\right)} \frac{W_{t}^{i}}{W_{t}^{1}}, \\
& \Longleftrightarrow \chi_{t+1}=\frac{\chi_{t}}{\alpha\left(1-\lambda_{1}\right)}-\sum_{i=1}^{n} \frac{\lambda_{i}}{\alpha\left(1-\lambda_{1}\right)} \frac{W_{t}^{i}}{W_{t}^{1}},
\end{aligned}
$$

where the second equation takes advantage of the definition (A.1). Completing then a further change of variables:

$$
\zeta_{t}:=\chi_{t}\left[\alpha\left(1-\lambda_{1}\right)\right]^{t},
$$

it is finally obtained that:

$$
\zeta_{t+1}=\zeta_{t}-\sum_{i=1}^{n} \lambda_{i} \frac{W_{t}^{i}}{W_{t}^{1}}\left[\alpha\left(1-\lambda_{1}\right)\right]^{t} .
$$

Making use of Equation (5a) considered for $i=1$ and $i \in\{2,3, \ldots, n\}$, it derives that the relative wealth with respect to the most patient agent states as:

$$
\begin{aligned}
& \frac{W_{t+1}^{i}}{W_{t+1}^{1}}=\frac{\left(1-\lambda_{i}\right) W_{t}^{i}}{\left(1-\lambda_{1}\right) W_{t+1}^{1}} \\
& \Longrightarrow \frac{W_{t}^{i}}{W_{t}^{1}}=\left(\frac{1-\lambda_{i}}{1-\lambda_{1}}\right)^{t} \frac{W_{0}^{i}}{W_{0}^{1}}
\end{aligned}
$$

Replacing then Equation (A.5) in Equation (A.4), it finally derives that:

$$
\zeta_{t+1}=\zeta_{t}-\sum_{i=1}^{n} \lambda_{i}\left[\alpha\left(1-\lambda_{i}\right)\right]^{t} \frac{W_{0}^{i}}{W_{0}^{1}} .
$$

Developing backward the R.H.S. of the preceding equation:

$$
\zeta_{t}=\zeta_{\mathrm{o}}-\sum_{i=1}^{n} \lambda_{i} \frac{W_{\mathrm{o}}^{i}}{W_{\mathrm{o}}^{1}} \sum_{\tau=0}^{t-1}\left[\alpha\left(1-\lambda_{i}\right)\right]^{\tau} .
$$

By definition, $\zeta_{t}$ must be non negative for every $t \in \mathbb{N}$ as $\chi_{t} \geqslant 0$ prevails for every $t \in \mathbb{N}$. As $\alpha\left(1-\lambda_{i}\right)<1$ for every $i \in\{1,2, \ldots, n\},\left(\zeta_{t}\right)_{t \in \mathbb{N}}$ converges to a limit $\zeta$ that is determined by Equation (A.6):

$$
\zeta=\zeta_{\mathrm{o}}-\sum_{i=1}^{n} \lambda_{i} \frac{W_{\mathrm{o}}^{i}}{W_{\mathrm{o}}^{1}} \frac{1}{1-\alpha\left(1-\lambda_{i}\right)} .
$$


Further, as $\zeta_{t} \geqslant 0$ for $t \in \mathbb{N}$, it derives that $\zeta \geqslant 0$.

Finally establishing that $\zeta=0$, assume, ad absurdum, that $\zeta>0$ does prevail. From the respective definitions of $\chi_{t}$ and $\zeta_{t}$, it derives that:

$$
\begin{aligned}
\zeta_{t} & =\frac{\left(K_{t}\right)^{\alpha}}{W_{t}^{1}}\left[\alpha\left(1-\lambda_{1}\right)\right]^{t} \longrightarrow \zeta>0 \quad \text { as } t \rightarrow+\infty, \\
& \Longleftrightarrow \frac{W_{t}^{1}}{\left(K_{t}\right)^{\alpha}} \sim \frac{1}{\zeta}\left[\alpha\left(1-\lambda_{1}\right)\right]^{t} \quad \text { as } t \rightarrow+\infty .
\end{aligned}
$$

It is therefore obtained that:

$$
\begin{aligned}
& \frac{W_{t}^{1}}{\left(K_{t}\right)^{\alpha}} \rightarrow 0 \text { as } t \rightarrow+\infty, \\
& \text { and } \begin{aligned}
\frac{W_{t}^{i}}{\left(K_{t}\right)^{\alpha}} & =\frac{W_{t}^{i}}{W_{t}^{1}} \frac{W_{t}^{1}}{\left(K_{t}\right)^{\alpha}} \\
& =\left(\frac{1-\lambda_{i}}{1-\lambda_{1}}\right)^{t} \frac{W_{0}^{i}}{W_{\mathrm{o}}^{1}} \frac{W_{t}^{1}}{\left(K_{t}\right)^{\alpha}} \rightarrow 0 \text { as } t \rightarrow+\infty .
\end{aligned}
\end{aligned}
$$

From Equation $\left(5^{b}\right)$, it is possible to write:

$$
K_{t+1}=\left(K_{t}\right)^{\alpha}\left[1-\sum_{i=1}^{n} \lambda_{i} \frac{W_{t}^{i}}{\left(K_{t}\right)^{\alpha}}\right] .
$$

But it has been established that $W_{t}^{i} /\left(K_{t}\right)^{\alpha} \rightarrow 0$ as $t \rightarrow+\infty$, so that the preceding equation in turn implies that $K_{t} \rightarrow \tilde{K}=1$ as $t \rightarrow+\infty$, for $\tilde{K}$ that is such that $\tilde{K}=(\tilde{K})^{\alpha}$, which is impossible as this corresponds to an overaccumulation configuration. The wealth of the agents would become infinite in conjunction with $\sum_{t=0}^{+\infty}\left(w_{t} / \prod_{\tau=0}^{t} R_{\tau}\right)=+\infty$, whence the proof that $\zeta=0$.

As $\zeta=0$, it is obtained that:

$$
\begin{aligned}
\zeta_{\mathrm{o}} & =\sum_{i=1}^{n} \lambda_{i} \frac{W_{\mathrm{o}}^{i}}{W_{\mathrm{o}}^{1}} \frac{1}{1-\alpha\left(1-\lambda_{i}\right)} \\
& =\sum_{i=1}^{n} \lambda_{i} \frac{W_{\mathrm{o}}^{i}}{W_{\mathrm{o}}^{1}} \sum_{\tau=0}^{+\infty}\left[\alpha\left(1-\lambda_{i}\right)\right]^{\tau} .
\end{aligned}
$$

Replacing the ensued expression for $\zeta_{\mathrm{o}}$ in Equation (A.6), it is obtained that:

$$
\begin{aligned}
\zeta_{t} & =\sum_{i=1}^{n} \lambda_{i} \frac{W_{\mathrm{o}}^{i}}{W_{\mathrm{o}}^{1}} \sum_{\tau=t}^{+\infty}\left[\alpha\left(1-\lambda_{i}\right)\right]^{\tau} \\
& =\sum_{i=1}^{n} \lambda_{i} \frac{W_{\mathrm{o}}^{i}}{W_{\mathrm{o}}^{1}} \frac{\left[\alpha\left(1-\lambda_{i}\right)\right]^{t}}{1-\alpha\left(1-\lambda_{i}\right)} .
\end{aligned}
$$

Making use of the respective definitions of $\chi_{t}$ and $\zeta_{t}$ :

$$
W_{t}^{1}=\frac{\left(K_{t}\right)^{\alpha}}{\zeta_{t}}\left[\alpha\left(1-\lambda_{1}\right)\right]^{t} .
$$

Taking advantage of Equation (A.5) of the relative wealths of the agents, it is further inferred that:

$$
W_{t}^{i}=\frac{W_{\mathrm{o}}^{i}}{W_{\mathrm{o}}^{1}} \frac{\left(K_{t}\right)^{\alpha}}{\zeta_{t}}\left[\alpha\left(1-\lambda_{i}\right)\right]^{t} .
$$

Eventually replacing Equations (A.7) and (A.8) in Equation (5b), it is derived that:

$$
\begin{aligned}
& K_{t+1}=\left(K_{t}\right)^{\alpha}\left(1-\gamma_{t}\right), \\
& \gamma_{t}:=\frac{\sum_{i=1}^{n} \lambda_{i} W_{\mathrm{o}}^{i}\left[\alpha\left(1-\lambda_{i}\right)\right]^{t}}{\sum_{i=1}^{n} \lambda_{i} W_{\mathrm{o}}^{i}\left[\alpha\left(1-\lambda_{i}\right)\right]^{t} /\left[1-\alpha\left(1-\lambda_{i}\right)\right]}
\end{aligned}
$$

and the details of the statement follow. 


\section{Proof of Proposition 3.1}

(i) Existence and uniqueness of the competitive equilibrium. Lemma 3.1 enables to determine the equilibrium dynamics up to the knowledge of the initial wealths

$$
W_{\mathrm{o}}^{i}=R a_{\mathrm{o}}^{i}+w_{\mathrm{o}}+\sum_{t=1}^{+\infty} \frac{w_{t}}{\prod_{\tau=1}^{t} R_{\tau}}
$$

with $a_{0}^{i}$ given such that $\sum_{i=1}^{n} a_{0}^{i}=K_{0}$. The expression of human wealth $\mu=w_{0}+\sum_{t=1}^{+\infty}\left[w_{t} / \prod_{\tau=1}^{t} R_{\tau}\right]$ remains unknown. Making use of $(4 a)$ and $(4 b)$, it is obtained that:

$$
\mu=\frac{1-\alpha}{n}\left[\left(K_{0}\right)^{\alpha}+\frac{K_{1}}{\alpha}+\frac{1}{\alpha} \sum_{t=2}^{+\infty} K_{t} \prod_{\tau=1}^{t-1}\left(\frac{\left(K_{\tau}\right)^{1-\alpha}}{\alpha}\right)\right],
$$

$\mu$ being thus an increasing function of $K_{0}, K_{1}, \ldots, K_{t}, \ldots$

Making use of (B.1), the equilibrium law of capital accumulation (6b) becomes:

$$
\begin{aligned}
& \gamma_{t}=\frac{\sum_{i=1}^{n} \lambda_{i} R_{0} a_{\mathrm{o}}^{i}\left[\alpha\left(1-\lambda_{i}\right)\right]^{t}+\mu \sum_{i=1}^{n} \lambda_{i}\left[\alpha\left(1-\lambda_{i}\right)\right]^{t}}{\sum_{i=1}^{n} \lambda_{i} R_{\mathrm{o}} a_{\mathrm{o}}^{i}\left[\alpha\left(1-\lambda_{i}\right)\right]^{t} /\left[1-\alpha\left(1-\lambda_{i}\right)\right]+\mu \sum_{i=1}^{n} \lambda_{i}\left[\alpha\left(1-\lambda_{i}\right)\right]^{t} /\left[1-\alpha\left(1-\lambda_{i}\right)\right]}, \\
& K_{t+1}=\left(K_{t}\right)^{\alpha}\left(1-\gamma_{t}\right) .
\end{aligned}
$$

Let $\mu$ be given. Taking advantage of (B.3), a sequence $\left(K_{t}(\mu)\right)_{t \in \mathbb{N}}$ is determined starting from the initial condition $K_{0}$. That sequence converges towards a value $\bar{K}$ that is independent of $\mu$ and such that $\bar{K}=\alpha(\bar{K})^{\alpha}\left(1-\lambda_{1}\right)$, whence $\alpha(\bar{K})^{\alpha-1}=1 /\left(1-\lambda_{1}\right)$. Defining then a function $Z(\mu)$ according to:

$$
Z(\mu):=\frac{1-\alpha}{n}\left[\left(K_{\mathrm{o}}\right)^{\alpha}+\frac{K_{1}(\mu)}{\alpha}+\frac{1}{\alpha} \sum_{t=2}^{+\infty} K_{t}(\mu) \prod_{\tau=1}^{t-1}\left(\frac{\left(K_{\tau}(\mu)\right)^{1-\alpha}}{\alpha}\right)\right] .
$$

The sequence $\left(K_{t}(\mu)\right)_{t \in \mathbb{N}}$ being convergent-the level $\bar{K}$ does indeed correspond to an underaccumulation steady state-, this function is well defined.

The existence of a competitive equilibrium is then available upon the existence of a value $\mu^{*}$ such that $Z\left(\mu^{*}\right)=\mu^{*}$, uniqueness being reached if and only if the function $Z(\cdot)$ assumes a unique fixed point.

Dealing first with the first argument, consider the two following sequences:

$$
\begin{aligned}
\gamma_{t}^{h} & :=\max \left\{\frac{\sum_{i=1}^{n} \lambda_{i} R_{\mathrm{o}} a_{\mathrm{o}}^{i}\left[\alpha\left(1-\lambda_{i}\right)\right]^{t}}{\sum_{i=1}^{n} \lambda_{i} R_{\mathrm{o}} a_{\mathrm{o}}^{i}\left[\alpha\left(1-\lambda_{i}\right)\right]^{t} /\left[1-\alpha\left(1-\lambda_{i}\right)\right]},\right. \\
\gamma_{t}^{\ell} & :=\min \left\{\frac{\sum_{i=1}^{n} \lambda_{i}\left[\alpha\left(1-\lambda_{i}\right)\right]^{t}}{\sum_{i=1}^{n} \lambda_{i} R_{0} a_{\mathrm{o}}^{i} \lambda_{i} \lambda_{i}\left[\alpha \left(1-\lambda_{0} a_{\mathrm{o}}^{i}\left[\alpha\left(1-\lambda_{i}\right)\right]^{t} /\left[1-\alpha\left(1-\lambda_{i}\right)\right]^{t} /\left[1-\alpha\left(1-\lambda_{i}\right)\right]\right.\right.}\right\} ; \\
& \left.\frac{\sum_{i=1}^{n} \lambda_{i}\left[\alpha\left(1-\lambda_{i}\right)\right]^{t}}{\sum_{i=1}^{n} \lambda_{i}\left[\alpha\left(1-\lambda_{i}\right)\right]^{t} /\left[1-\alpha\left(1-\lambda_{i}\right)\right]}\right\} .
\end{aligned}
$$

The coefficient $\gamma_{t}$ as given by (B.3) emerges as a monotone function of $\mu$, is enclosed between the values associated to $\mu=0$ and $\mu=+\infty$, whence, and for any $t \in \mathbb{N}$, the satisfaction of:

$$
\gamma_{t}^{\ell} \leqslant \gamma_{t} \leqslant \gamma_{t}^{h}
$$


Let then the sequences $\left(\bar{K}_{t}(\mu)\right)_{t \in \mathbb{N}}$ and $\left(\underline{K}_{t}(\mu)\right)_{t \in \mathbb{N}}$ respectively defined by:

$$
\begin{aligned}
& \bar{K}_{\mathrm{o}}=K_{0} ; \forall t \in \mathbb{N}, \bar{K}_{t+1}=\left(\bar{K}_{t}\right)^{\alpha}\left(1-\gamma_{t}^{\ell}\right) ; \\
& \underline{K}_{0}=K_{0} ; \forall t \in \mathbb{N}, \underline{K}_{t+1}=\left(\underline{K}_{t}\right)^{\alpha}\left(1-\gamma_{t}^{h}\right) .
\end{aligned}
$$

It is then inferred that,

$$
\begin{aligned}
& \forall t \in \mathbb{N}, \underline{K}_{t} \leqslant K_{t}(\mu) \leqslant \bar{K}_{t}, \\
& \lim _{t \rightarrow+\infty} \underline{K}_{t}=\lim _{t \rightarrow+\infty} \bar{K}_{t}=\lim _{t \rightarrow+\infty} K_{t}(\mu)=\bar{K},
\end{aligned}
$$

as $\lim _{t \rightarrow+\infty} \gamma_{t}^{\ell}=\lim _{t \rightarrow+\infty} \gamma_{t}^{h}=\lim _{t \rightarrow+\infty}=1-\alpha\left(1-\lambda_{1}\right)$. Let then in turn the coefficients $\underline{\mu}$ and $\bar{\mu}$ be defined as:

$$
\begin{aligned}
& \underline{\mu}=\frac{1-\alpha}{n}\left[\left(K_{0}\right)^{\alpha}+\frac{\underline{K}_{1}}{\alpha}+\frac{1}{\alpha} \sum_{t=2}^{+\infty} \underline{K}_{t} \prod_{\tau=1}^{t-1}\left(\frac{\left(\underline{K}_{\tau}\right)^{1-\alpha}}{\alpha}\right)\right], \\
& \bar{\mu}=\frac{1-\alpha}{n}\left[\left(K_{0}\right)^{\alpha}+\frac{\bar{K}_{1}}{\alpha}+\frac{1}{\alpha} \sum_{t=2}^{+\infty} \bar{K}_{t} \prod_{\tau=1}^{t-1}\left(\frac{\left(\bar{K}_{\tau}\right)^{1-\alpha}}{\alpha}\right)\right] .
\end{aligned}
$$

For every $\mu \geqslant 0, \mu \leqslant Z(\mu) \leqslant \bar{\mu}$ is hence satisfied. When considered on the interval $[\mu, \bar{\mu}]$, the function $Z(\mu)$ corresponds to a continuous function defined over a compact set. Indeed, the convergence of the sum defining $Z(\mu)$ is uniform:

$$
\begin{aligned}
& \sup _{(\mu)}\left[\frac{1-\alpha}{n} \frac{1}{\alpha} \sum_{t=T}^{+\infty} K_{t}(\mu) \prod_{\tau=1}^{t-1}\left(\frac{\left(K_{\tau}(\mu)\right)^{1-\alpha}}{\alpha}\right)\right] \leqslant \frac{1-\alpha}{n} \frac{1}{\alpha} \sum_{t=T}^{+\infty} \bar{K}_{t} \prod_{\tau=1}^{t-1}\left(\frac{\left(\bar{K}_{\tau}\right)^{1-\alpha}}{\alpha}\right) \\
& \text { with } \lim _{T \rightarrow+\infty} \frac{1-\alpha}{n} \frac{1}{\alpha} \sum_{t=T}^{+\infty} \bar{K}_{t} \prod_{\tau=1}^{t-1}\left(\frac{\left(\bar{K}_{\tau}\right)^{1-\alpha}}{\alpha}\right)=0 .
\end{aligned}
$$

The function $Z(\mu)$ being continuous over the compact set $[\mu, \bar{\mu}]$, it assumes a fixed point.

Focusing then on the uniqueness argument, assume, ad absurdum, that there exists two solutions $\mu_{1}$ and $\mu_{2}$ to the equation $Z(\mu)=\mu$, with $\mu_{1}<\mu_{2}$. The argument will proceed by showing that the solution sequences would then be such that $K_{t}\left(\mu_{1}\right)>K_{t}\left(\mu_{2}\right)$ for any $t \in \mathbb{N}$, that cannot be reconciled with the monotony of $K_{t}(\mu)$ and will establish uniqueness.

By recurrence, it is first established that:

$$
W_{\mathrm{o}}^{i}=R_{\mathrm{o}} a_{\mathrm{o}}^{i}+\mu_{1} \quad \text { and } \quad W_{\mathrm{o}}^{i^{\prime}}=R_{\mathrm{o}} a_{\mathrm{o}}^{i}+\mu_{2},
$$

whence and for every $i \in\{1, \ldots, n\}$, the obtention of $W_{0}^{i}<W_{0}^{i^{\prime}}$.

Letting then $\chi_{0}^{i}=W_{0}^{i} /\left(K_{0}\right)^{\alpha}$ and $\chi_{0}^{i^{\prime}}=W_{0}^{i^{\prime}} /\left(K_{0}\right)^{\alpha}$, it derives that, for every $i \in\{1, \ldots, n\}$, $\chi_{\mathrm{o}}^{i}<\chi_{\mathrm{o}}^{i^{\prime}}$ or, further,

$$
\gamma_{\mathrm{o}} \equiv \frac{\sum_{i=1}^{n} \lambda_{i} W_{\mathrm{o}}^{i}}{\left(K_{\mathrm{o}}\right)^{\alpha}}<\frac{\sum_{i=1}^{n} \lambda_{i} W_{\mathrm{o}}^{i^{\prime}}}{\left(K_{\mathrm{o}}\right)^{\alpha}} \equiv \gamma_{\mathrm{o}}^{\prime}
$$

It remains to establish that, for every $i \in\{1, \ldots, n\}, \chi_{1}^{i}<\chi_{1}^{i^{\prime}}$ and $\gamma_{1}<\gamma_{1}^{\prime}$. For that purpose, first consider the ratio of equations $(5 a)$ and $(5 b)$ when considered at date $t=0$ :

$$
\begin{gathered}
\chi_{1}^{i} \equiv \frac{W_{1}^{i}}{\left(K_{1}(\mu)\right)^{\alpha}}=\frac{\alpha\left(1-\lambda_{i}\right) W_{0}^{i}}{\left(K_{0}\right)^{\alpha}\left(1-\gamma_{\mathrm{o}}\right)}=\alpha\left(1-\lambda_{i}\right) \frac{\chi_{\mathrm{o}}^{i}}{1-\gamma_{\mathrm{o}}}, \\
\chi_{1}^{i^{\prime}} \equiv \frac{W_{1}^{i^{\prime}}}{\left(K_{1}(\mu)\right)^{\alpha}}=\frac{\alpha\left(1-\lambda_{i}\right) W_{\mathrm{o}}^{i^{\prime}}}{\left(K_{\mathrm{o}}\right)^{\alpha}\left(1-\gamma_{\mathrm{o}}^{\prime}\right)}=\alpha\left(1-\lambda_{i}\right) \frac{\chi_{0}^{i_{0}^{\prime}}}{1-\gamma_{\mathrm{o}}^{\prime}} .
\end{gathered}
$$


But, and as for every $i \in\{1, \ldots, n\}, \chi_{0}^{i}<\chi_{0}^{i^{\prime}}$ and $\gamma_{0}<\gamma_{0}^{\prime}$, it naturally follows that $\chi_{1}^{i}<\chi_{1}^{i^{\prime}}$ for every $i \in\{1, \ldots, n\}$, whence, eventually, the satisfaction of

$$
\gamma_{1}=\sum_{i=1}^{n} \lambda_{i} \chi_{1}^{i}<\sum_{i=1}^{n} \lambda_{i} \chi_{1}^{i^{\prime}}=\gamma_{1}^{\prime}
$$

By recurrence, assume that, for every $i \in\{1, \ldots, n\}, \chi_{t}^{i}<\chi_{t}^{i^{\prime}}$ and $\gamma_{t}<\gamma_{t}^{\prime}$ : it remains to establish that for every $i \in\{1, \ldots, n\}, \chi_{t+1}^{i}<\chi_{t+1}^{i^{\prime}}$ and $\gamma_{t+1}<\gamma_{t+1}^{\prime}$. For that intend, considering again the ratio of equations $\left(5^{a}\right)$ and $\left(5^{b}\right)$, it is obtained that:

$$
\begin{aligned}
& \chi_{t+1}^{i}=\frac{\alpha\left(1-\lambda_{i}\right) \chi_{t}^{i}}{1-\gamma_{t}}, \\
& \chi_{t+1}^{i^{\prime}}=\frac{\alpha\left(1-\lambda_{i}\right) \chi_{t}^{i^{\prime}}}{1-\gamma_{t}^{\prime}} .
\end{aligned}
$$

Whence the holding of $\chi_{t+1}^{i}<\chi_{t+1}^{i^{\prime}}$ and

$$
\gamma_{t+1}=\sum_{i=1}^{n} \lambda_{i} \chi_{t+1}^{i}<\sum_{i=1}^{n} \lambda_{i} \chi_{t+1}^{i^{\prime}}=\gamma_{t+1}^{\prime}
$$

It is thus established that $\gamma_{t}<\gamma_{t}^{\prime}$ for all $t \in \mathbb{N}$. From equation $(6 b)$, it is inferred that, for every $t \in \mathbb{N}, K_{t}\left(\mu_{1}\right)>K_{t}\left(\mu_{2}\right)$. The expression of $Z(\mu)$ being however increasing as a function of any of the $K_{t}(\mu)$, it is eventually obtained that:

$$
Z\left(\mu_{1}\right)>Z\left(\mu_{2}\right)
$$

a contradiction.

(ii) a/ From Equation $(6 b)$, the sequence $\left(K_{t}\right)_{t \in \mathbb{N}}$ converges to a steady state $\bar{K}$ such that $\bar{K}=$ $(K)^{\alpha} \alpha\left(1-\lambda_{1}\right)$.

b/ From Equation $(6 a)$, it derives that:

$$
\begin{aligned}
\lim _{t \rightarrow+\infty} W_{t}^{1} & =\frac{\left[1-\alpha\left(1-\lambda_{1}\right)\right]}{\lambda_{1}}(\bar{K})^{\alpha}, \\
\lim _{t \rightarrow+\infty} W_{t}^{i} & =0 \text { for every } i \neq 1 .
\end{aligned}
$$

c/ Making use of Equations ( $6 a)$ and of the consumption decision rule (3), it is derived that:

$$
\begin{aligned}
\lim _{t \rightarrow+\infty} c_{t}^{1} & =\left[1-\alpha\left(1-\lambda_{1}\right)\right](\bar{K})^{\alpha}, \\
\lim _{t \rightarrow+\infty} c_{t}^{i} & =0 \text { for every } i \neq 1 .
\end{aligned}
$$

The details of the statement follow.

\section{Proof of Lemma 4.1}

Consider an agent with the preference parameters $(\beta, \delta)$. As for Lemma 3.1, the following variables are used:

$$
\begin{aligned}
X_{t} & =\frac{W_{t}}{\Pi_{t}} \\
x_{t} & =\frac{c_{t}}{\Pi_{t}}
\end{aligned}
$$


with $\Pi_{t}$ defined as:

$$
\begin{gathered}
\Pi_{0}=1 \\
\text { for } t \geq 1, \Pi_{t}=\prod_{\tau=1}^{t} R_{\tau}
\end{gathered}
$$

For a linear technology, $R_{\tau}=A$ and $\Pi_{t}=A^{t}$. The intertemporal budget constraint (1) is $X_{t+1}=$ $X_{t}-x_{t}$ with $x_{t} \leq X_{t}$.

With a CES utility function, the objective function of self $t(2)$ can be written as :

$$
\frac{\left(\delta A^{(\sigma-1) / \sigma}\right)^{t}}{(\sigma-1) / \sigma}\left\{\left(x_{t}\right)^{(\sigma-1) / \sigma}+\beta \sum_{\tau=1}^{+\infty}\left(\delta A^{(\sigma-1) / \sigma}\right)^{\tau}\left(x_{t+\tau}\right)^{(\sigma-1) / \sigma}\right\}
$$

Consider the game defined on a $T$ period horizon. The variables $X_{t}$ and $x_{t}$ are now denoted by $X_{t}^{T}$ and $x_{t}^{T}$. For $t \leq T$, the $t$-self objective function is proportional to:

$$
\frac{1}{(\sigma-1) / \sigma}\left\{\left(x_{t}^{T}\right)^{(\sigma-1) / \sigma}+\beta \sum_{\tau=1}^{T-t}\left(\delta A^{(\sigma-1) / \sigma}\right)^{\tau}\left(x_{t+\tau}^{T}\right)^{(\sigma-1) / \sigma}\right\}
$$

For notational simplicity: $\Delta=\left(\delta A^{(\sigma-1) / \sigma}\right)$. The game is solved by backward induction. As the objective functions of all selves are homothetic, it is straightforward that the solution will take the form:

$$
x_{t}^{T}=\lambda_{t}^{T} X_{t}^{T}
$$

For $t=T$, the solution is obvious: $\lambda_{T}^{T}=1$. Assuming that the game has been solved till period $t+1$, one obtains, $\forall \tau$ with $2 \leq \tau \leq T$,

$$
x_{t+\tau}^{T}=\lambda_{t+\tau}^{T}\left(1-\lambda_{t+\tau-1}^{T}\right) \ldots\left(1-\lambda_{t+1}^{T}\right) X_{t+1}^{T}
$$

and $x_{t+1}^{T}=\lambda_{t+1}^{T} X_{t+1}^{T}$. Replacing in the $t$-self objective function, we get:

$$
\begin{aligned}
& \frac{1}{(\sigma-1) / \sigma}\left\{\left(x_{t}^{T}\right)^{(\sigma-1) / \sigma}+\beta \Delta\left(X_{t+1}^{T}\right)^{(\sigma-1) / \sigma} Z_{t}^{T}\right\}, \\
& \text { with } Z_{t}^{T}=\left(\lambda_{t+1}^{T}\right)^{(\sigma-1) / \sigma}+\Delta\left(\lambda_{t+2}^{T}\right)^{(\sigma-1) / \sigma}\left(1-\lambda_{t+1}^{T}\right)^{(\sigma-1) / \sigma} \\
& \qquad+\cdots+\Delta^{T-t-1}\left(\lambda_{T}^{T}\right)^{(\sigma-1) / \sigma}\left(1-\lambda_{T-1}^{T}\right)^{(\sigma-1) / \sigma} \ldots\left(1-\lambda_{t+1}^{T}\right)^{(\sigma-1) / \sigma} .
\end{aligned}
$$

The program of self $t$ can be written:

$$
\begin{aligned}
\max _{\left(x_{t}^{T}, X_{t+1}^{T}\right)} & \frac{1}{(\sigma-1) / \sigma}\left\{\left(x_{t}^{T}\right)^{(\sigma-1) / \sigma}+\beta \Delta\left(X_{t+1}^{T}\right)^{(\sigma-1) / \sigma} Z_{t}^{T}\right\} \\
\text { s.t. } & x_{t}^{T}+X_{t+1}^{T}=X_{t}^{T} .
\end{aligned}
$$

The solution is $x_{t}^{T}=\lambda_{t}^{T} X_{t}^{T}, X_{t+1}^{T}=\left(1-\lambda_{t}^{T}\right) X_{t}^{T}$, with

$$
\lambda_{t}^{T}=\frac{1}{1+(\beta \Delta)^{\sigma}\left(Z_{t}^{T}\right)^{\sigma}}
$$

From the definition of $Z_{t}^{T}$, one gets:

$$
Z_{t}^{T}=\left(\lambda_{t+1}^{T}\right)^{(\sigma-1) / \sigma}+\Delta\left(1-\lambda_{t+1}^{T}\right)^{(\sigma-1) / \sigma} Z_{t+1}^{T}
$$


Using (6) in $t$ and $t+1$ to eliminate $Z_{t}^{T}$ and $Z_{t+1}^{T}$ in (7), it is obtained that:

$$
\frac{1}{\beta \Delta}\left(\frac{1-\lambda_{t}^{T}}{\lambda_{t}^{T}}\right)^{1 / \sigma}=\left(\lambda_{t+1}^{T}\right)^{(\sigma-1) / \sigma}+\Delta\left(1-\lambda_{t+1}^{T}\right)^{(\sigma-1) / \sigma} \frac{1}{\beta \Delta}\left(\frac{1-\lambda_{t+1}^{T}}{\lambda_{t+1}^{T}}\right)^{1 / \sigma}
$$

After some simplifications,

$$
\lambda_{t}^{T}=\frac{1}{1+\Delta^{\sigma}\left[1-(1-\beta) \lambda_{t+1}^{T}\right]^{\sigma} / \lambda_{t+1}^{T}}
$$

Define

$$
f(\lambda)=\frac{1}{1+\Delta^{\sigma}[1-(1-\beta) \lambda]^{\sigma} / \lambda}
$$

From backward induction with $\lambda_{T}^{T}=1$, all the sequence $\lambda_{t}^{T}$ is defined as: $\lambda_{t}^{T}=f^{T-t}(1)$.

The function $f$ has the following properties: $f(0)=0, f$ increasing on $[0,1], f^{\prime}(0)=1+(1-\beta) \sigma>1$. $f$ has two fixed points, $o$ and $\lambda^{*}$, which is solution of:

$$
\Delta^{\sigma}\left[1-(1-\beta) \lambda^{*}\right]^{\sigma}=1-\lambda^{*}
$$

From these properties, if $T \rightarrow+\infty$, we get for the limit strategy:

$$
\lim _{T \rightarrow+\infty} \lambda_{t}^{T}=\lim _{T \rightarrow+\infty} f^{T-t}(1)=\lambda^{*},
$$

which establishes the statement.

QED

\section{E. Proof of Proposition 4.1}

(i) The obtention of equations $(9 a)$ and $(9 b)$ is immediate.

The obtention of the coefficient $\gamma_{t}$ proceeds as follows. First, take advantage of $(9 a)$ :

$$
\frac{W_{t}^{i}}{W_{t}^{1}}=\left(\frac{1-\lambda_{i}}{1-\lambda_{1}}\right)^{t} \frac{a_{0}^{i}}{a_{0}^{1}}
$$

Parallely make use of $\left(5^{a}\right)$ and $\left(5^{b}\right)$. It derives that:

$$
\begin{aligned}
& \frac{K_{t+1}}{W_{t+1}^{1}}=\frac{A K_{t}}{A\left(1-\lambda_{1}\right) W_{t}^{1}}-\sum_{i=1}^{n} \frac{\lambda_{i} W_{t}^{i}}{A\left(1-\lambda_{1}\right) W_{t}^{1}} \\
& \Longleftrightarrow \frac{K_{t+1}}{W_{t+1}^{1}}=\frac{K_{t}}{\left(1-\lambda_{1}\right) W_{t}^{1}}-\frac{1}{A\left(1-\lambda_{1}\right)} \sum_{i=1}^{n} \lambda_{i}\left(\frac{1-\lambda_{i}}{1-\lambda_{1}}\right)^{t} \frac{a_{0}^{i}}{a_{0}^{1}} .
\end{aligned}
$$

Letting $\zeta_{t}:=\left(1-\lambda_{1}\right)^{t} K_{t} / W_{t}^{1}$, Equation (E.1) reformulates to:

$$
\zeta_{t+1}=\zeta_{t}-\frac{\left(1-\lambda_{1}\right)^{t}}{A} \sum_{i=1}^{n} \lambda_{i}\left(\frac{1-\lambda_{i}}{1-\lambda_{1}}\right)^{t} \frac{a_{0}^{i}}{a_{0}^{1}} .
$$

Forward develop Equation (E.2):

$$
\begin{aligned}
\zeta_{t} & =A^{-1} \sum_{i=1}^{n} \lambda_{i} \frac{a_{0}^{i}}{a_{0}^{1}} \sum_{\tau=t}^{+\infty}\left(1-\lambda_{i}\right)^{\tau} \\
& =A^{-1} \sum_{i=1}^{n} \lambda_{i} \frac{a_{0}^{i}}{a_{0}^{1}} \frac{\left(1-\lambda_{i}\right)^{t}}{\lambda_{i}} \\
& =A^{-1} \sum_{i=1}^{n} \frac{a_{0}^{i}}{a_{0}^{1}}\left(1-\lambda_{i}\right)^{t} .
\end{aligned}
$$


It is eventually obtained that:

$$
\begin{aligned}
\frac{W_{t}^{1}}{K_{t}} & =\frac{\left(1-\lambda_{1}\right)^{t}}{\zeta_{t}} \\
& =\frac{A\left(1-\lambda_{1}\right)^{t}}{\sum_{i=1}^{n}\left(1-\lambda_{i}\right)^{t} a_{\mathrm{o}}^{i} / a_{\mathrm{o}}^{1}} \\
\frac{W_{t}^{i}}{K_{t}} & =\frac{\left(1-\lambda_{i}\right)^{t}}{\left(1-\lambda_{1}\right)^{t}} \frac{a_{\mathrm{o}}^{i}}{a_{\mathrm{o}}^{1}} \frac{W_{t}^{1}}{K_{t}} \\
& =\frac{A\left(1-\lambda_{i}\right)^{t} a_{\mathrm{o}}^{i}}{\sum_{j=1}^{n}\left(1-\lambda_{j}\right)^{t} a_{\mathrm{o}}^{j}} .
\end{aligned}
$$

Whence:

$$
\begin{aligned}
\gamma_{t} & =\frac{\sum_{i=1}^{n} \lambda_{i} W_{t}^{i}}{A K_{t}} \\
& =\frac{\sum_{i=1}^{n} \lambda_{i}\left(1-\lambda_{i}\right)^{t} a_{\mathrm{o}}^{i}}{\sum_{i=1}^{n}\left(1-\lambda_{i}\right)^{t} a_{\mathrm{o}}^{i}} .
\end{aligned}
$$

(ii) This is immediate from the expression of $\gamma_{t}$.

\section{F. Proof of Proposition 4.2}

Let $\chi_{i}:=1-\lambda_{i}$. From Equation (8), it is defined from the following equation:

$$
A^{1-1 / \sigma}\left(\chi_{i}\right)^{1 / \sigma}=\delta_{i}\left[\beta_{i}+\chi_{i}\left(1-\beta_{i}\right)\right] .
$$

Consider then the two ensued straight lines equations $\xi_{i}(\chi)=\delta_{i}\left[\beta_{i}+\chi\left(1-\beta_{i}\right)\right], i=1,2$. These two lines assume an intersection at $(\hat{\chi}, \hat{\xi})$, for $\hat{\xi} \in] \mathrm{o}, 1[$. Its first coordinate is indeed readily computed to:

$$
\hat{\chi}=\frac{\delta_{1} \beta_{1}-\delta_{2} \beta_{2}}{\delta_{2}\left(1-\beta_{2}\right)-\delta_{1}\left(1-\beta_{1}\right)},
$$

where $\hat{\chi}>0$ is ensured under the assumptions of the proposition. Further noticing that:

$$
\begin{aligned}
\hat{\chi}<1 & \Longleftrightarrow \delta_{1} \beta_{1}-\delta_{2} \beta_{2}<\delta_{2}\left(1-\beta_{2}\right)-\delta_{1}\left(1-\beta_{1}\right) \\
& \Longleftrightarrow \delta_{1}<\delta_{2} .
\end{aligned}
$$

while the second coordinate is readily computed to:

$$
\hat{\xi}=\frac{\delta_{1} \delta_{2}\left(\beta_{1}-\beta_{2}\right)}{\delta_{2}\left(1-\beta_{2}\right)-\delta_{1}\left(1-\beta_{1}\right)} .
$$

Consider now the function $\Upsilon(\chi):=A^{1 / \sigma-1} \chi^{1 / \sigma}$. It is then noticed that $\Upsilon(0)$ is lower that both of the straight-lines $\xi_{i}(\mathrm{o})=\delta_{i} \beta_{i}, i \in\{1,2\}$. In opposition to this, $\Upsilon(1)$ is greater than $\xi_{i}(1)=\delta_{i}, i \in\{1,2\}$. Indeed and under Assumption 1, any of the straight-lines is to assume a unique intersection point with $\Upsilon(\chi)$ within the interval ]o, $1[$.

Finally define $\hat{A}$ from the holding of $(\hat{A})^{1 / \sigma-1}(\hat{\chi})^{1 / \sigma}=\hat{\xi}$.

- For $A<\hat{A}$, one obtains

$$
A^{1 / \sigma-1}(\hat{\chi})^{1 / \sigma}>(\hat{A})^{1 / \sigma-1}(\hat{\chi})^{1 / \sigma}
$$

whence the holding of $\left.\chi_{1}, \chi_{2} \in\right] \mathrm{o}, \hat{\chi}\left[\right.$ and the satisfaction of $\chi_{1}>\chi_{2}$, that implies $\lambda_{1}<\lambda_{2}$. 
- For $A>\hat{A}$, one oppositely obtains

$$
A^{1 / \sigma-1}(\hat{\chi})^{1 / \sigma}<(\hat{A})^{1 / \sigma-1}(\hat{\chi})^{1 / \sigma},
$$

whence the holding of $\left.\chi_{1}, \chi_{2} \in\right] \hat{\chi}, 1\left[\right.$ and the satisfaction of $\chi_{1}<\chi_{2}$, that implies $\lambda_{1}>\lambda_{2}$.

The statement follows.

QED

\section{G. Proof of Proposition $4 \cdot 3$}

Consider a naïve agent with parameters $(\beta, \delta)$. For a naïve self deciding at period $\mathrm{o}$, all of the decisions starting from date o do correspond to the ones taken by an agent with a discount factor of $\delta$, i.e., without any biais and for $\beta=1$. The corresponding value for the coefficient $\lambda$ is hence available as:

$$
\lambda=1-\delta^{\sigma} A^{\sigma-1} .
$$

For every $t \geqslant 1$, the law of evolution of his wealth and the level of his consumption would be available as:

$$
\begin{aligned}
& W_{t+1}=A(1-\lambda) W_{t}, \\
& c_{t}=\lambda W_{t} .
\end{aligned}
$$

The associated value of the objective by date $t=0$ hence derives as:

$$
\frac{\left(c_{0}\right)^{1-1 / \sigma}}{1-1 / \sigma}+\frac{\beta}{1-1 / \sigma}(\lambda A)^{1-1 / \sigma}\left(W_{0}-c_{0}\right)^{1-1 / \sigma}\left\{\sum_{\tau=1}^{+\infty} \delta^{\tau}[A(1-\lambda)]^{(\tau-1)(1-1 / \sigma)}\right\} .
$$

The first-order condition of the optimisation problem is available as:

$$
\left(c_{0}\right)^{-1 / \sigma}=\beta(\lambda A)^{1-1 / \sigma} \frac{\left(W_{0}-c_{0}\right)^{-1 / \sigma} \delta}{1-\delta[A(1-\lambda)]^{1-1 / \sigma}} .
$$

From this equation, the $c_{0}$ solution assumes a form $c_{0}=\lambda$ and

$$
\begin{aligned}
& \left(\lambda^{n}\right)^{-1 / \sigma}=\frac{\beta \delta\left[\left(1-(\delta)^{\sigma} A^{\sigma-1}\right) A\right]\left(1-\lambda^{n}\right)^{-1 / \sigma}}{1-\delta\left[\delta^{\sigma} A^{\sigma}\right]^{1-1 / \sigma}} \\
& \lambda^{n}=\frac{1-\delta^{\sigma} A^{\sigma-1}}{1-\delta^{\sigma} A^{\sigma-1}\left(1-\beta^{\sigma}\right)} .
\end{aligned}
$$

In order to compare $\lambda$ and $\lambda^{n}$, let $\chi:=1-\lambda$ and $\chi^{n}:=1-\lambda^{n}$. The coefficient $\chi$ is defined as the solution of:

$$
\chi=\delta^{\sigma} A^{\sigma-1}[\beta+(1-\beta) \chi]^{\sigma},
$$

that is to be compared with:

$$
\chi^{n}=\frac{\delta^{\sigma} A^{\sigma-1} \beta^{\sigma}}{1-\delta^{\sigma} A^{\sigma-1}\left(1-\beta^{\sigma}\right)} .
$$

This leads to:

$$
\chi^{n}>\chi \Longleftrightarrow \chi^{n}>\delta^{\sigma} A^{\sigma-1}\left[\beta+(1-\beta) \chi^{n}\right]^{\sigma} .
$$


Having completed a range of simplifications, it is eventually obtained that the satisfaction of this inequation reformulates as the one of:

$$
\left[1-\delta^{\sigma} A^{\sigma-1}\left(1-\beta^{\sigma}\right)\right]^{\sigma-1}>\left[1-\delta^{\sigma} A^{\sigma-1}\left(1-\beta^{\sigma-1}\right)\right]^{\sigma} .
$$

Consider the function $G(\cdot)$ defined by:

$$
G(\beta)=(\sigma-1) \ln \left[1-\delta^{\sigma} A^{\sigma-1}\left(1-\beta^{\sigma}\right)\right]-\sigma \ln \left[1-\delta^{\sigma} A^{\sigma-1}\left(1-\beta^{\sigma-1}\right)\right] .
$$

From the previous results, $\chi>\chi^{n} \Leftrightarrow G(\beta)>0$. Further noticing that $G(1)=0$, the derivative of $G(\cdot)$ is available as:

$$
\begin{aligned}
D G(\beta) & =\sigma(\sigma-1)\left[\frac{\delta^{\sigma} A^{\sigma-1} \beta^{\sigma-1}}{1-\delta^{\sigma} A^{\sigma-1}\left(1-\beta^{\sigma}\right)}-\frac{\delta^{\sigma} A^{\sigma-1} \beta^{\sigma-2}}{1-\delta^{\sigma} A^{\sigma-1}\left(1-\beta^{\sigma-1}\right)}\right] \\
& =\sigma(\sigma-1) \delta^{\sigma} A^{\sigma-1} \beta^{\sigma-2}\left[\frac{\beta}{1-\delta^{\sigma} A^{\sigma-1}\left(1-\beta^{\sigma}\right)}-\frac{1}{1-\delta^{\sigma} A^{\sigma-1}\left(1-\beta^{\sigma-1}\right)}\right] \\
& =\sigma(\sigma-1) \delta^{\sigma} A^{\sigma-1} \beta^{\sigma-2}\left[\frac{(\beta-1)\left(1-\delta^{\sigma} A^{\sigma-1}\right)}{\left[1-\delta^{\sigma} A^{\sigma-1}\left(1-\beta^{\sigma}\right)\right]\left[1-\delta^{\sigma} A^{\sigma-1}\left(1-\beta^{\sigma-1}\right)\right]}\right]
\end{aligned}
$$

Then, and for $\beta<1, D G(\beta)<0$ if $\sigma>1$ while $D G(\beta)>0$ if $\sigma<1$. As $G(1)=0$, it is obtained that, for $\beta<1$ :

$$
\begin{cases}\chi^{n}>\chi & \text { if } \sigma>1 \\ \chi^{n}<\chi & \text { if } \sigma<1\end{cases}
$$

As a consequence:

$$
\begin{cases}\lambda^{n}<\lambda & \text { if } \sigma>1 \\ \lambda^{n}>\lambda & \text { if } \sigma<1 .\end{cases}
$$

The statement follows. 